\title{
Effect of Carbon Forms on Properties of Ag-C Composites Contact Materials
}

\author{
Katarzyna PIETRZAK ${ }^{1}$, Krystyna FRYDMAN ${ }^{1}$, Danuta WÓJCIK-GRZYBEK ${ }^{1}$ *, \\ Andrzej GLADKI ${ }^{1}$, Anna BANKKOWSKA ${ }^{1}$, Piotr BORKOWSKI ${ }^{2}$ \\ ${ }^{1}$ Institute of Electronic Materials Technology, 133 Wólczyńska Str., 01-919 Warsaw, Poland \\ ${ }^{2}$ Lodz University of Technology, Department of Electrical Apparatus; 18/22 B. Stefanowskiego Str., 90-924 Lodz, Poland \\ crossref http://dx.doi.org/10.5755/j01.ms.24.1.17769
}

Received 16 March 2017; accepted 05 June 2017

\begin{abstract}
This paper presents the manufacturing method of silver based composite materials containing $3 \%$ vol. carbon forms (nanotubes and graphene). The most significant challenge was to obtain good dispersion of carbon in the metallic matrix. The applying of suitable dispersants allows to get uniform distribution of carbon reinforcement. Triton X-100 and ultrasonic support were used in the powder mixing process. Ag-nanotubes and Ag-graphene contact tips were made using Spark Plasma Sintering process (SPS). The results of research into both physical and electrical properties of these composites are presented. It has been demonstrated that the form of introduced carbon exerts influence on the electrical characteristics of contacts, and particularly on arc erosion. Performed electrical test indicated that Ag-nanotubes contacts showed higher resistance to arc erosion than Ag-graphene contacts. It can be explained by the better dispersion to individual carbon nanotubes their lower than for graphene edge defects density and due to this higher thermal and electrical conductivity.

Keywords: composite materials, silver-nanotubes, silver-graphene, arc erosion, contact resistance.
\end{abstract}

\section{INTRODUCTION}

Silver-based composites are known as a material group finding wide application in commonly used low-voltage air contactors. Complex phenomena accompanying current flow in electrical contacts help distinguish a group of essential features, typical of contact materials. One can enumerate here:

- high electrical and thermal conductivity;

- low and stable electrical resistance of contact couples - contact resistance;

- high welding and abrasion resistance;

- high mobility of discharging channels in relation to the surface of contacts.

Low-voltage air contactors work under various conditions, depending on their future area of application. A universal contact material meeting all of the above mentioned requirements at the same time does not exist. In addition, contact materials used in air connectors are expected to be resistant to environmental factors at raised temperatures.

Above all, they have to show resistance to corrosion, typically increasing their electrical resistance, which in consequence leads to higher working temperature and more heat released by the electrical current. That is why, making use of composite materials with a silver matrix may turn out to be an effective solution.

It has to be highlighted though that the high thermal and electrical conductivity of silver does not compensate for its insufficient resistance to thermal erosion, inextricably linked with its low melting point, and, in consequence, tendency to weld. This results from the intense thermodynamic activity of silver's surface, even at temperatures as low as $450 \mathrm{~K}$ [1]. That is why, in the case of silver-based contact materials,

\footnotetext{
${ }^{*}$ Correspoding author. Tel.: +48 22 639059059; fax: +48 228645496 . E-mail address: danuta.grzybek@itme.edu.pl (D. Wójcik-Grzybek)
}

additional phases are introduced with the aim of enhancing their switching properties. Depending on the target of application the different reinforcing phases are used in silver based composite contact materials: metal oxides $\left(\mathrm{SnO}_{2}\right.$, $\mathrm{Fe}_{2} \mathrm{O}_{3}, \mathrm{ZnO}$ ), refractory phases (W, Mo, WC), metals (Ni, $\mathrm{Fe}$ ) and carbon [2]. For a long time, Ag-C composites containing 3-5\% wt. of carbon have been frequently used in electrical air switches $[3,4]$. They are characterized by very high resistance to welding and relatively low ohm contact resistance. Because of these properties, such composites are mainly used in contacts of residual-current devices and in main contacts of high current switches. A disadvantage of $\mathrm{Ag}-\mathrm{C}$ composites is their low resistance to arc erosion.

Carbon, most frequently used in the form of graphite, is such a reinforcing phase. It occurs in a number of allotropic variations, including graphite, diamond, fullerenes, nanotubes and such amorphous forms as carbon black or glassy carbon. An interesting question to pose is whether the application of carbon in an allotropic form other than graphite has influence on the switching properties of $\mathrm{Ag}-\mathrm{C}$ composites, and particularly on their resistance to arc erosion. So far, the relationship between switching properties and the size of graphite particles [2-4], the array of particles (fibres) in relation to the contact's active surface $[5,6]$, and microstructure dependent on the production method $[7,8]$ have been studied. In addition, research into using different forms of carbon in the Ag-C5 composite [9] and impact of the percentage of nanotubes in the Ag-C5 composite on physical properties [10] has been carried out; however, it has not been focused on the switching properties of contacts. The most recent scientific investigation has shown beneficial properties of materials when used as the reinforcing phase of carbon in the form of graphene and nanotubes [11, 12]. One atom-thick graphene surface is flexible, strong and transparent. It has very high thermal conductivity $(4800-5300 \mathrm{~W} / \mathrm{mK})$ and 
low electrical resistance. Carbon nanotubes are strong and stiff materials. CNTs can withstand pressure up to $25 \mathrm{GPa}$ without deformation and the bulk modulus of super hard phase nanotubes is up to $546 \mathrm{GPa}$. Thermal conductivity along axis measurement at room temperature is about $3500 \mathrm{~W} / \mathrm{mK}$ [13]. Due to unique properties researchers are looking for their applications in new materials. It is expected the introduction of nanosized forms of carbon (nanotubes or graphene) in a metallic matrix would have the potential to improve the mechanical, thermal and electrical properties.

Composite contact materials, including Ag-C-type composites, are most frequently obtained using powder metallurgy methods and the way they are formed is chosen taking into account specific chemical composition. For silver-based composites with a reinforcing phase in the form of carbon, i.e. graphite, diamond or glassy carbon, a three-stage formation process, including pressing, sintering and repressing $[2,3,14]$, is usually selected. Ag-C contact materials having density close to the theoretical value are produced using spark plasma sintering (SPS) [15]. This method entails the uniaxial pressing of powder in a conductive die and simultaneous heating of the system through the passage of the electrical current. Due to its mechanical properties and electrical conduction, graphite is the most often used material in the production of dies. The current flowing through a die and powder causes strong Joule heat generation, responsible for heating of the entire system. A quick sintering process using the SPS method is attainable as a result of insignificant thermal capacity of the system. This is particularly important when aiming at obtaining a material with high density and restricting growth of grains $[13,16]$.

This paper presents the production method of Ag-nanotubes and Ag-graphene composite powders, from which contacts were made using Spark Plasma Sintering (SPS). In addition, the results of research into both arc erosion and contact resistance for these materials are shown.

\section{EXPERIMENTAL PROCEDURE}

In this work, the analysed Ag-C contact materials with reinforcing phases in the form of multi-wall nanotubes and graphene flakes were obtained using the SPS technique. Dendritic silver powder with the particle size of $10 \mu \mathrm{m}$, manufactured by the Innovator company (Gliwice), Aldrich nanotubes measuring 5-9 $\mu \mathrm{m}, \varphi=110-170 \mathrm{~nm}$ (MWNTs), and SkySpring Nanomaterials, Inc. graphene nanopowder (GNP) with the flake size of $1-5 \mu \mathrm{m}$ have been used. The scanning Zeiss AURIGA electron microscope was used to determine powder morphology and samples microstructures. Observations were conducted in secondary electrons mode at primary electrons energy in the range of 1 to $20 \mathrm{kV}$. Fig. 1 presents powder morphology, whereas Fig. $1 \mathrm{~b}$ shows that dry nanotubes create balls, composed of tangled, single forms. Graphene flakes appear as compact structures, forming particles reaching the size of several microns (see Fig. $1 \mathrm{c}$ ).

Therefore, a key issue when it comes to the production of homogenous $\mathrm{Ag}-\mathrm{C}$ composites is the appropriate dispersion of carbon forms, separating single nanotubes and graphene flakes.

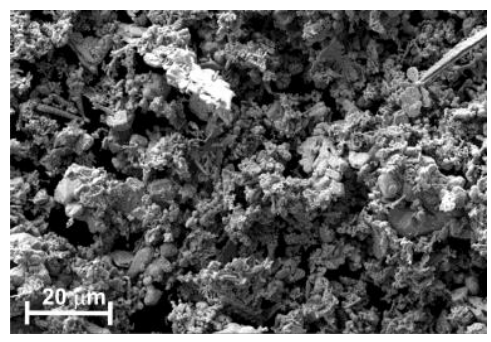

a

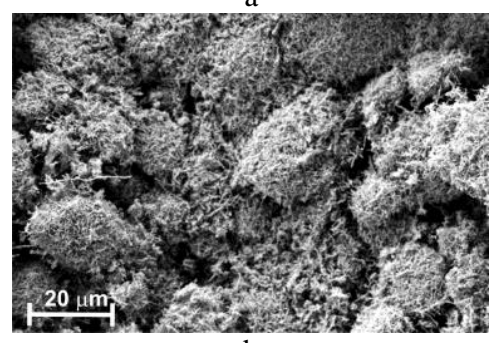

b

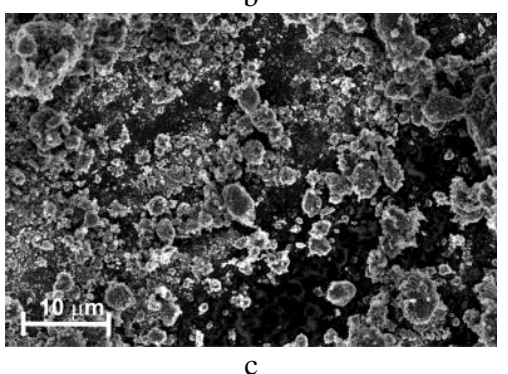

Fig. 1. Powder morphology: $a-A g ; b-M W N T s ; c-G N P$

Dispersion of graphene nanostructures is most frequently carried out in aqueous solutions of various dispersants $[17,18]$. The choice of dispersants depends on the composite material's designation and its purity. The following, surface-active chemical compounds [17] prove to be the most efficient dispersants:

- Dimethylformamide (DMF);

- N-Methyl-2-pyrrolidone (NMP);

- Triton X-100.

All three surfactants appear in the form of liquids freely soluble in water. DMF and NMP particles are polar, with electrostatic interactions observed between them and dispersed materials. Triton $\mathrm{X}-100\left(\mathrm{C}_{14} \mathrm{H}_{22} \mathrm{O}\left(\mathrm{C}_{2} \mathrm{H}_{4} \mathrm{O}\right)_{n}\right)$ is a nonionic surfactant that has a hydrophilic polyethylene oxide chain and an aromatic hydrocarbon lipophilic or hydrophobic group. Triton $\mathrm{X}-100$ is widely used in different applications including reducing surface tension of aqueous solutions and dispersion of carbon materials for soft composite materials. It is a good micellar catalyst. Not only do micelles significantly reduce the surface tension of solutions, but also dynamically interact with the solid phase, thus decreasing forces binding carbon forms together to produce balls or clusters. As a result of preliminary attempts to separate nanotubes using the above mentioned dispersants, Triton X-100 turned out to be the most suitable surface-active compound.

Nanotube dispersion was a multi-step process. Individual stages included: 
- multi-step sonication of Triton X-100 liquid solutions with nanotubes;

- centrifugation of solutions lasting a predefined period of time to obtain a homogeneous nanotube fraction;

- vacuum filtering of the centrifuged solutions through a chosen $\mathrm{Ag}$ powder portion placed on a $0.2 \mu \mathrm{m}$ filter;

- drying of the composite powder at $323 \mathrm{~K}$.

The selection of devices used in all of the presented steps, together with process parameters, is shown in Fig. 2.

\section{PROCESSES}

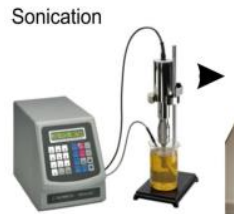

Spinning Vacuum filtration

Cycle:1secON/1secOFF Time:4 min Energy: $147 \mathrm{~J}$

Fig. 2. Sonication, separation and FC filtration system: a-VCX500 ultrasonic homogenizer; b-centrifuge MPW-351 system; c-millipore vacuum filtering system with a $0.2 \mu \mathrm{m}$ filter

Duration of both sonication and centrifugation has been determined based on absorption spectra from the Thermo ELECTRON CORPORATION Helios $\gamma$ spectrophotometer and especially the $180-340 \mathrm{~nm}$ wave range, for which Rayleigh dissipation was expected for particles measuring less than the dissipated wave length. The maximal absorption peak indicated the optimal time (Fig. 3).

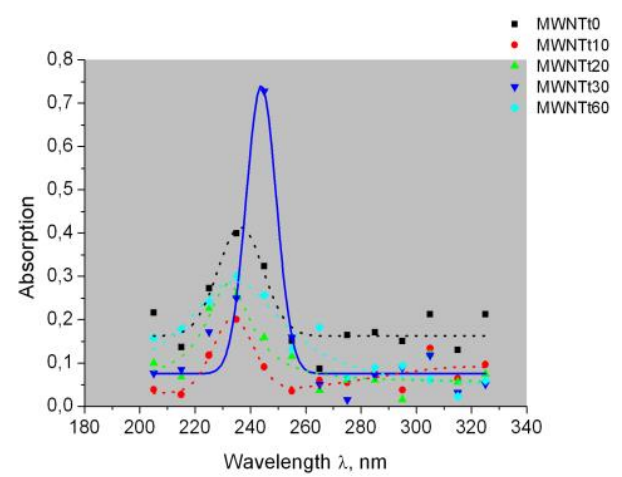

a

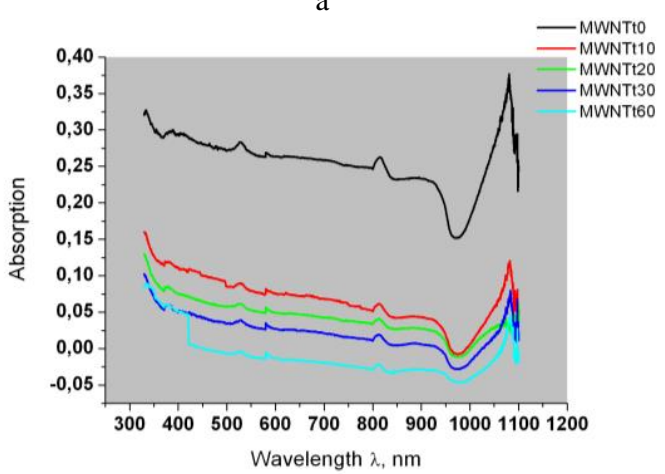

b

Fig. 3. a-short-wave lengths spectrum of absorption light for solution with MWNTs in various centrifugation times; $\mathrm{b}$-full spectrum for absorption in various centrifugation times
Transmission spectra from the spectrophotometer were used to estimate the weight of nanotubes found in solutions after centrifugation. Average transmission values for the sonicated solution with the known initial weight of nanotubes and average transmission values after centrifugation proved to be useful as well. The LambertBeer law has enabled determination of the relative mass fraction in the centrifuged solution in relation to the noncentrifuged solution with the known nanotube mass. For optimal sonication and centrifugation times, the LambertBeer absorbance ratio $[19,20]$ equalled 0.7 irrespective of the type of carbon forms (FC) and the $0.1 \%-30 \%$ range of the analyzed FC concentration. For instance, this means that for instance for each initial weight unit of nanotubes (MWNTs) seven parts remained in the solution, whereas three parts were deposited on the sidewalls of the container used to perform centrifugation.

The described production method of Ag-C composite powder proved appropriate for obtaining nanotubes (MWNTs). In the case of graphene nanopowder (GNP), a considerable portion of graphene flakes easily passed through a layer of powder and a filter during vacuum infiltration. That is why, ethanol was used as dispersion liquid. The solution was sonicated and centrifuged, mixed in ultrasounds with $\mathrm{Ag}$ powder directly after, and dried.

The composite contact material was obtained using the SPS method. Fig. 4 presents the scheme of SPS process and photograph of ITME SPS device used to prepare of samples. Ag-C powder mixtures were placed in a graphite die. The electrical current passes through the graphite elements of the system and heats the powders (both Ag and $\mathrm{C}$ are good conductors of electricity) as the effect of Joule's heating. The following sintering conditions (after preliminary tests) were chosen: temperature $-1123 \mathrm{~K}$, time - 15 minutes, pressure $-50 \mathrm{MPa}$. The heating rate was $100^{\circ} \mathrm{C} / \mathrm{min}$ (decreased to $20^{\circ} \mathrm{C} / \mathrm{min}$ in the final stage), that allowed in the fast and repetitive way for obtaining almost fully dense materials.

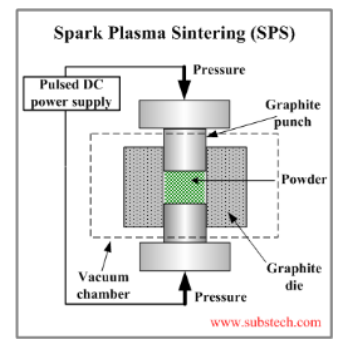

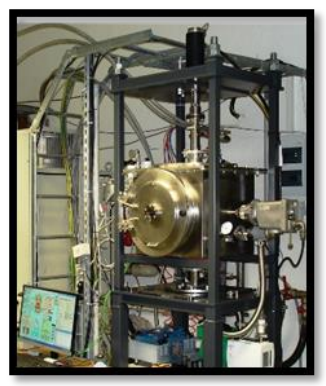

b

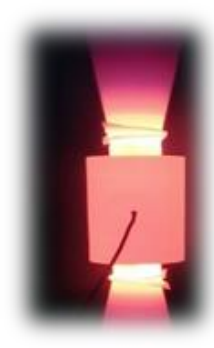

Fig. 4. a-SPS process scheme [www.subtech.com]; b-ITME SPS device; $c$ - sintering of sample 
The contact tips were prepared from sintered samples by mechanical machining to final dimensions $\phi=8 \mathrm{~mm}$ $\mathrm{h}=1 \mathrm{~mm}$. On such samples density and hardness measurement were made. For parts of samples intended for electrical tests, onto the assembly surface of the contacts, the layer of $0.2 \mathrm{~mm}$ thick $\mathrm{AgCu} 28$ binder, enable welding to a copper substrate, was applied. Good contact between the electrode material and the substrate has an important effect on the materials behaviour under operation conditions [21].

Electrical tests included arc erosion and contact resistance measurements under model test conditions (Table 1) [22].

Table 1. Test conditions

\begin{tabular}{|l|c|c|}
\hline \multicolumn{1}{|c|}{ Test parameter } & Unit & Value \\
\hline Mechanical parameters: & $\mathrm{mm}$ & 8 \\
Contact diameter & $\mathrm{mm}$ & 5 \\
Contact gap & $\mathrm{mm}$ & 2 \\
Contact tilt & $\mathrm{N}$ & 10 \\
Contact force & $\mathrm{N}$ & 30 \\
Opening spring force & & \\
\hline Electrical parameters: & $\mathrm{V}, \mathrm{Hz}$ & 230,50 \\
Voltage supply & $\mathrm{A}$ & 60 \\
Current (peak value) & $\mathrm{deg}$ & 1 \\
Contact opening phase angle & $\mathrm{op} / \mathrm{h}$ & 1200 \\
Operating frequency & & \\
\hline
\end{tabular}

\section{RESULTS AND DISCUSSION}

The SEM analysis confirmed the good separation of MWNTs and GNP. Fig. 5 presents SEM images, showing the morphology of the following composite materials: $\mathrm{Ag}+3 \%$ vol. nanotubes and $\mathrm{Ag}+3 \%$ vol. graphene. Clear separation of nanotubes in $\mathrm{Ag}$ powder (Fig. $5 \mathrm{a}$ ) and excellent dispersion of graphene in $\mathrm{Ag}$ powder can be noticed (Fig. 5 b).
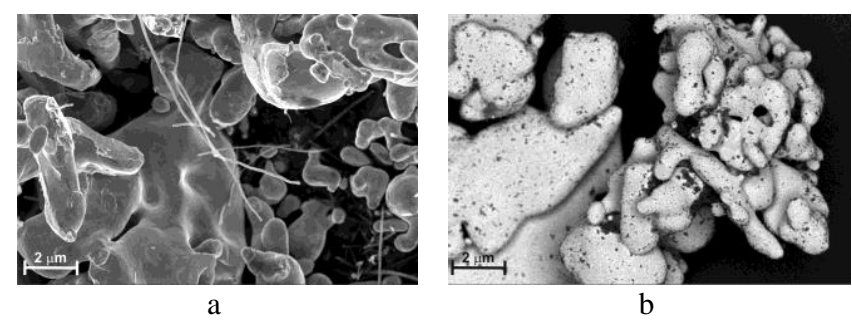

Fig. 5. Morphology of composite powders: $\mathrm{a}-\mathrm{Ag}+3 \%$ vol. MWNTs; b-Ag $+3 \%$ vol. GNP

SEM images of the outcome contact materials fracture can be found in Fig. 6.

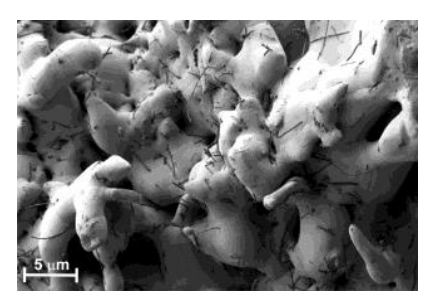

a

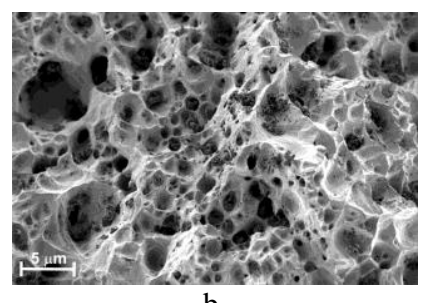

b
Fig. 6. Structure of samples fracture after sintering: $\mathrm{a}-\mathrm{Ag}+3 \%$ vol. MWNTs; $\mathrm{b}-\mathrm{Ag}+3 \%$ vol. GNP
The performed analysis of these SEM images indicates that:

- there is clear separation of nanotubes and their homogeneous distribution in the silver matrix Fig. 6 a);

- graphene nanopowder was dispersed to form small, nanometre-size objects (Fig. 6 b).

Density of the sintered samples was measured by the method based on Archimedes' law, whereas their hardness was measured with the Struers DuraScan hardness tester, following the Vickers method and using the load of $9.81 \mathrm{~N}$. Theoretical density was defined based on the volumetric content of graphene or nanotubes in the composite, assuming that the theoretical density of silver is $\gamma_{\mathrm{Ag}}=10.5 \mathrm{gcm}^{-3}$ and that of nanotubes or graphene is $\gamma_{C}=1.22 \mathrm{gcm}^{-3}$. The estimated theoretical density of the $\mathrm{Ag}-3 \%$ vol. C composite equals $\gamma_{\text {theor }}=10.22 \mathrm{gcm}^{-3}$. Both density and hardness values for the contact composites are collected in Table 2.

Table 2. Physical properties of composites

\begin{tabular}{|c|c|c|c|}
\hline \multirow[b]{2}{*}{ Materials } & \multicolumn{2}{|c|}{ Density SPS process after } & \multirow[b]{2}{*}{$\begin{array}{c}\text { Hardness, } \\
\text { HV1 }\end{array}$} \\
\hline & $\begin{array}{l}\text { Real density, } \\
\mathrm{g} / \mathrm{cm}^{3}\end{array}$ & $\begin{array}{l}\text { Relative density, } \\
\% \text { of theoretical } \\
\text { density }\end{array}$ & \\
\hline $\begin{array}{c}\mathrm{Ag}+3 \% \text { vol. } \\
\text { MWNTs }\end{array}$ & 9.83 & 96.18 & 41 \\
\hline $\begin{array}{c}\mathrm{Ag}+3 \% \text { vol. } \\
\text { GNP }\end{array}$ & 9.97 & 97.55 & 54 \\
\hline
\end{tabular}

Despite good distribution of the reinforcing phase and SPS method use to samples sintering density has not been reached the theoretical density level. The presence of pores at the $\mathrm{Ag} / \mathrm{C}$ interface constitutes a barrier to the flow of heat and electricity.

The contact life is used to determine the number of switching steps made during arc operation under established test conditions. The more switching cycles the contacts withstand, the more probable is the long contact life of the contactors in which they will be used. Most of all, this confirms the high quality of the composite material.

The arc erosion results are presented in Fig. 7. Values marked with the $\Delta m$ symbol in Table 3 (black colour in Fig. 7) were obtained experimentally. Based on these, lognormal statistical distribution estimators, i.e. $\Delta m_{\mathrm{M}}$ median and $\Delta m_{95}$ values in Table 3 and cross-hatched areas in Fig. 7, were estimated.

Table 3. Average $\Delta \underline{m}_{\mathrm{M}}$ and forecasted $\Delta m_{95}$ mass decrement values for examined contacts, $\mathrm{mg}$

\begin{tabular}{|c|c|c|c|c|}
\hline \multirow{2}{*}{$\begin{array}{c}\text { Mass } \\
\text { decrement }\end{array}$} & \multicolumn{2}{|c|}{50.000} & \multicolumn{2}{c|}{100.000} \\
\cline { 2 - 5 } & Ag-GNP & $\begin{array}{c}\text { Ag- } \\
\text { MWNTs }\end{array}$ & Ag-GNP & $\begin{array}{c}\text { Ag- } \\
\text { MWNTs }\end{array}$ \\
\hline$\Delta m_{95}$ & 107.8 & 68.3 & 217.9 & 123.7 \\
\hline$\Delta m_{\mathrm{M}}$ & 59.3 & 34.3 & 93.3 & 62.8 \\
\hline
\end{tabular}

They are forecasts with a $5 \%$ error, which means extreme mass decrements of individual contacts. Contacts' mass decrement is taken as a base quantity in the 
assessment of their erosion resistance. All analyzed silverbased contacts proved to be resistant to electric arc; however, those with the nanotube addition are more resistant $(\sim 40 \%)$ than the contacts with graphene. The mean mass decrement value after 100 thousand switching cycles equalled $\Delta m_{\mathrm{M}}=62.8 \mathrm{mg}$ for $\mathrm{Ag}+$ MWNTs contacts and $\Delta m_{\mathrm{M}}=93.3 \mathrm{mg}$ for Ag-GNP contacts.

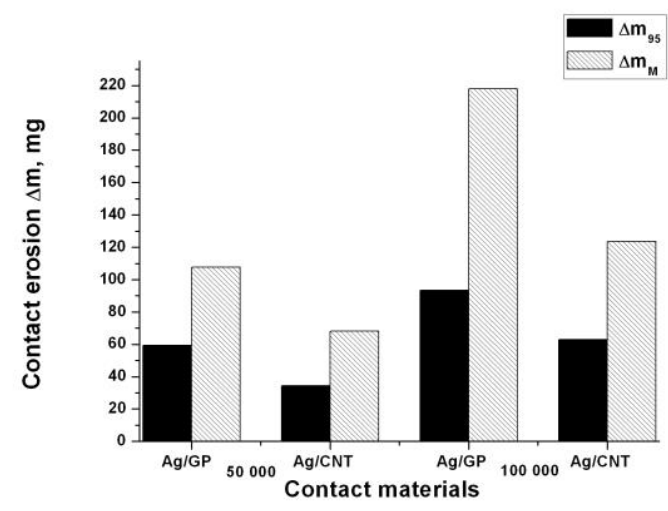

Fig. 7. Results of arc erosion tests after 50 and 100 thousand switching cycles

Electrical analyses carried out for these materials in low-voltage electrical contacts showed that by comparison with the reference material, which are always pure silver contacts (Ag 99.9\%) having similar dimensions [23], the Ag-nanotubes material was characterized by higher resistance to the erosive activity of the electric arc. All experiments were conducted using both identical test equipment and current values.

The contact resistance measurements were performed during switching cycles of Ag-MWNTs and Ag-GNP composites. The obtained results are presented in Fig. 8.

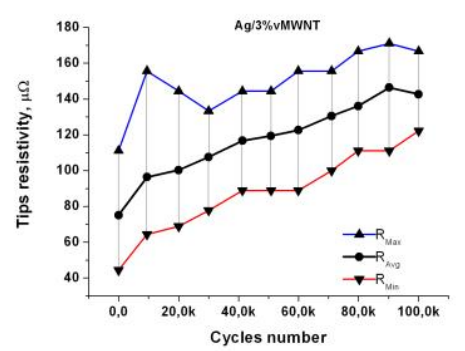

a

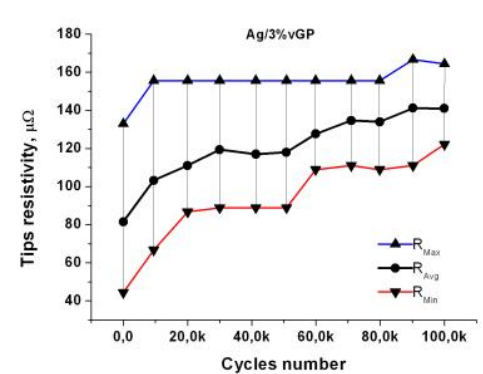

b

Fig. 8. Contact resistance values worked out during contact erosion measurements after 100 thousand switching cycles for: a-Ag-MWNTs; $b$ - Ag-GNP composites

The research showed that the average resistance values for silver-based contacts at first grow rapidly by around
$50 \%$ of their initial value, next, after more than 100 thousand switching cycles they start to increase slowly, with a steadier growth observable for Ag-nanotubes contacts. The final mean resistance value for the contacts based on silver reached around $140 \mu \Omega$. Therefore, as a result of electric arc activity after 100 thousand switching cycles, contact resistance for the analyzed material increased only two times. These are not spectacular growths, but still considerable since, they exert influence on the temperature increase in the operated contacts and should be taken into account when constructing contact systems.

After selected contacts had worked in electric arc and after 100 thousand switching cycles, metallographic specimen was made to carry out morphological analysis of the near-surface layer created as a result of electric arc activity, using the Zeiss AURIGA scanning electron microscope (Fig. 9).
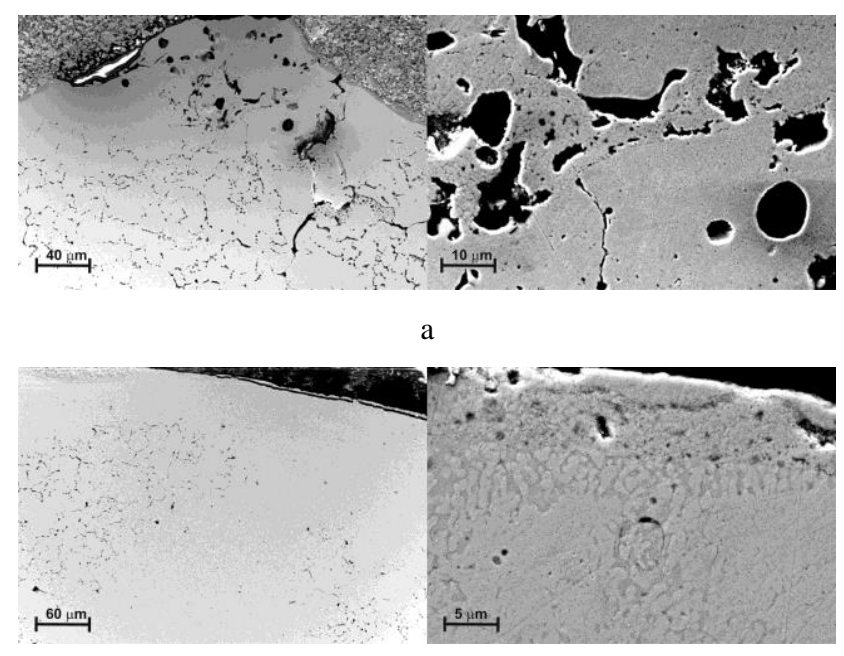

$\mathrm{b}$

Fig. 9. SEM image of contacts in cross section after operation in electric arc: $a-A g-G N P ; b-A g-M W N T s$

The microstructural analysis showed that erosion process is similar, irrespective of the form of carbon used. The near-surface layer appears to be highly irregular and is alternately composed of areas with carbon and without it. In addition, one can see cracks and numerous pores, most probably formed during the escape of $\mathrm{CO}$ and $\mathrm{CO}_{2}$ gases created due to reaction between carbon and atmospheric oxygen as well as when arc-melted silver solidifies.

The electrical test revealed the essential operating differences of the contact tips made of silver-reinforced GP and MWNTs. The comparison of mass losses with the contact structure the tests after, identifies the differences in the heat dissipation, generated by electrical current flowing through the material. Thermal conductivity is, among other things, the inverse proportional function of structural edge defects, in this case, carbon forms.

Carbon nanotubes are rated to $1 \mathrm{D}$ materials, and GP flakes to 2D dimension. Accepting the same degree of both phases fragmentation, the 1D structure will always have less boundary defects than 2D. The reinforced phases used in the research were different in size. The number of significantly smaller than nanotubes GP objects had to be several times greater in the same $3 \%$ vol. Thus, the 
number of carbon defects in Ag3\% vol. GP had to be significantly higher than in the case of MWNTs using. This fact was related to differences in the thermal conductivity of both composites and apparent differences in structure and contact mass losses after electrical tests.

\section{CONCLUSIONS}

Based on the performed research it can be concluded:

1. The most significant issue when producing $\mathrm{Ag}$ nanotubes (MWNTs) and Ag-graphene (GNP) composites is adequate dispersion of carbon forms (MWNTs and GNP) in the silver matrix. Good dispersion was achieved by application TritonX-100 dispersant and ultrasonic support.

2. Analysis of composites structures indicates for clear separation and homogeneous distribution of nanotubes and excellent dissipation of graphene nanopowder in the Ag matrix.

3. Uniform distribution of carbon forms in the silver matrix has impact on their physical and electrical properties.

4. Ag-nanotubes contacts after 100 thousand switching cycles showed higher resistance to arc erosion (average mass decrement value $\Delta m_{\mathrm{M}}=62.8 \mathrm{mg}$ ) than Ag-graphene contacts $\left(\Delta m_{\mathrm{M}}=93.3 \mathrm{mg}\right)$. It can be explained by less-defected structure of metal-carbon interface in the case of using carbon nanotubes.

5. The optimisation of sintering process should be provided to limit materials porosity and improve thermal and electrical properties of composite materials.

\section{Acknowledgments}

The results presented in this paper were obtained as part of the "GRAMCOM" project (Contract No Graftech/NCBiR/10/29/2013 with the National Centre for Research and Development) within the framework of the GRAF-TECH program.

\section{REFERENCES}

1. Fergus, J.W., Mallipedi, C.V.S., Edwards, D. Silver/SilverOxide Composite Coating for Intrinsically Adaptive Thermal Regulation Composites Part B 29R 1998: pp. 51-56. https://doi.org/10.1016/S1359-8368(97)00010-3

2. Slade, P. Electrical Contacts: Principles and Applications Marcel Dekker, Inc., New York, 1999: pp. 741-749.

3. Keil, A., Merl, W.A., Vinaricky, E. Elektrische Kontakte und ihre Werkstoffe; Springer, Berlin, 1984: pp. 195-196.

4. Wingert, P. The Effects of Interrupting Elevated Currents on the Erosion and Structure of Silver-Graphite 42th IEEE Holm Conference with $18^{\text {th }}$ ICEC, Chicago, 1996: pp. 60-69.

5. Behrens, V., Mahle, E., Michal, R., Saeger, K.E. Ein neues Silber/Graphit-Kontaktmaterial auf der Basis von Graphitfasern Metallwissenschaft und Technik 47 (7) 1993: pp. 639-643.

6. Behrens, V., Honig, Th., Kraus, A., Mahle, E., Michal, R., Saeger, K.E. The Results of Different Silver/Graphite Contact Materials in Regard to Applications in Circuit Breakers $41^{\text {st }}$ IEEE Holm Conference 1995: pp. 393-397. https://doi.org/10.1109/HOLM.1995.482896

7. Peuker, C., Hauner, F. Influence of Microstructure on the Switching Behaviour of AgC SAP, Lodz, 2001: pp. 213-218.
8. Wieser, H., Hauner, F. The Thermo-Mechanical Cracking of Agc Contacts with Parallel Carbon Structure $21^{\text {st }}$ ICEC, Zurich, 2002: pp. 438-442.

9. Jiang, P., Li, F., Wang, Y. Effect of Different Types of Carbon on Microstructure and Arcing Behavior of $\mathrm{Ag} / \mathrm{C}$ Contact Materials IEEE Transactions on Components and Packaging Technologies 29 (2) 2006: pp. 420-423. https://doi.org/10.1109/TCAPT.2006.875873

10. Feng, Y., Yuan, H.L., Zhang, M. Fabrication and Properties of Silver Matrix Composites Reinforced by Carbon Nanotubes Materials Characterization 55 2005: pp. 211-218. https://doi.org/10.1016/j.matchar.2005.05.003

11. Pasricha, R., Gupta, S., Srivastava, A.K. A Facile and Novel Synthesis of Ag-Graphene-Based Nanocomposites www.small-journal.com, 2009.

12. Wang, S., Wang, S., Xie, M., Liu, A., Hu, J. Effect of Graphene Content on Microstructure and Properties of Multilayer Graphene/Silver Composite Precious Metals 37 (2) 2016: pp. $51-56$.

13. Pietrzak, K., Sobczak, N., Chmielewski, M., Homa, M., Gazda, A., Zybała, R., Strojny-Nędza, A. Effects of Carbon Allotropic Forms on Microstructure and Thermal Properties of $\mathrm{Cu}-\mathrm{C}$ Composites Produced by SPS Journal of Materials Engineering and Performance 25 (8) 2016: pp. $3077-3083$

https://doi.org/10.1007/s11665-015-1851-0

14. Borkowski, P., Walczuk, E., Wójcik-Grzybek, D., Frydman, K., Zasada, D. Electrical Properties of Ag-C Contact Materials Including Different Carbon Allotropic Form 25th ICEC\&56th IEEE Holm Conference on Electrical Contacts Charleston 2010: pp. 167-175.

15. Marek, I., Vojtech, D., Michalcova, A., Kubalik, T.F. Highstrength Bulk Nano-Crystalline Silver Prepared by Selective Leaching, Combined with Spark Plasma Sintering Materials Science \& Engineering A 627 2015: pp. 326-332. https://doi.org/10.1016/j.msea.2015.01.014

16. Wejrzanowski, T., Grybczuk, M., Chmielewski, M., Pietrzak, K., $\quad$ Kurzydlowski, K.J., $\quad$ Strojny-Nędza, A. Thermal Conductivity of Metal-Graphene Composites Materials \& Design 99 2016: pp. 163-173. https://doi.org/10.1016/j.matdes.2016.03.069

17. Guardia, L. Fernández-Merino, M.J., Paredes, J.I., SolísFernández, P., Villar-Rodil, S., Martínez-Alonso, A. Tascón, J.M.D. High-Throughput Production of Pristine Graphene in an Aqueous Dispersion Assisted by Non-Ionic Surfactants Carbon 49 2011: pp. 1653-1662. https://doi.org/10.1016/j.carbon.2010.12.049

18. Wang, J. Früchtl, D., Blau, W.J. The Importance of Sol Rent for Optical Milling of Carbon Nanotubes Dispersions Optics Communications 283 2010: pp. 464-468.

19. Haken, H., Wolf, Ch.W. The Physics of Atoms and Quanta Springer 2005: pp. 399-415.

20. Theopanides, T. Infrared Spectroscopy - Materials Science, Engineering and Technology InTech Europe 2012: pp. 213-232. https://doi.org/10.5772/2055

21. Pietrzak, K., Olesińska, W., Kaliński, D., Strojny-Nędza, A. The Relationship Between Microstructure and Mechanical Properties of Directly Bonded Copper-Alumina Ceramics Joints Bulletin of the Polish Academy of Sciences Technical Sciences 62 (1) 2014: pp. 1-10. https://doi.org/10.2478/bpasts-2014-0003

22. Borkowski, P., Boczkowski, D., Wysocki, T. ComputerControlled System for Testing Contacts on Switching High Current Measurement 40 2007: pp. 94-299. https://doi.org/10.1016/j.measurement.2006.05.017

23. Walczuk, E. Arc Erosion of High Current Contacts in the Aspect of CAD of Switching Devices 38th IEEE Holm Conference on Electrical Contacts, Philadelphia 1992: pp. $1-16$.

https://doi.org/10.1109/HOLM.1992.246940 